\title{
Stars and brown dwarfs in the $\sigma$ Orionis cluster: the Mayrit catalogue ${ }^{\star}$
}

\author{
J. A. Caballero ${ }^{\star \star}$ \\ Max-Planck-Institut für Astronomie, Königstuhl 17, 69117 Heidelberg, Germany \\ e-mail: caballero@astrax.fis.ucm.es \\ Received 15 May 2007 / Accepted 22 October 2007

\begin{abstract}
Context. The young $\sigma$ Orionis cluster is an indispensable basis for understanding the formation and evolution of stars, brown dwarfs, and planetary-mass objects. Our knowledge of its stellar population is, however, incomplete.

Aims. I present the Mayrit catalogue, which comprises most of the stars and high-mass brown dwarfs in the cluster.

Methods. The basis of this work is an optical-near infrared correlation between the 2MASS and DENIS catalogues in a circular area of radius 30 arcmin centred on the OB-type binary $\sigma$ Ori AB. The analysis is supported by a bibliographic search of confirmed cluster members with features of youth and by additional X-ray, mid-infrared, and astrometric data.

Results. I list $241 \sigma$ Orionis stars and brown dwarfs with known features of youth, 97 candidate cluster members (40 are new), and 115 back- and foreground sources in the survey area. The 338 cluster members and member candidates constitute the Mayrit catalogue.

Conclusions. This catalogue is a suitable input for studying the spatial ditribution, multiplicity, properties and frequency of discs, and the complete mass function of $\sigma$ Orionis.
\end{abstract}

Key words. astronomical data bases: miscellaneous - stars: low-mass, brown dwarfs -

Galaxy: open clusters and associations: individual: $\sigma$ Orionis

\section{Introduction}

The $\sigma$ Orionis cluster in the Ori OB $1 \mathrm{~b}$ association is getting as important for the study of the formation, evolution, and characterisation of stars and substellar objects as other famous clusters and star-forming regions, like the Hyades, the Pleiades, the Orion Nebula Cluster, or the Taurus-Auriga complex. The $\sigma$ Orionis cluster is young $(3 \pm 2 \mathrm{Ma})$, nearby $(d \sim 385 \mathrm{pc})$, and relatively free of extinction (Lee 1968; Brown et al. 1994; Oliveira et al. 2002; Zapatero Osorio et al. 2002a; Sherry et al. 2004; Béjar et al. 2004b; Caballero 2007d). First identified by Garrison (1967) and Lyngå (1981), $\sigma$ Orionis was rediscovered by Wolk (1996) and Walter et al. (1997). They reported a clustering of young low-mass stars, many of them positionally coincident with X-ray sources, surrounding the Trapezium-like, multiple stellar system $\sigma$ Ori in the vicinity of the Horsehead Nebula (see Caballero 2007b, for a description of the multiple system $\sigma$ Ori and its surroundings). Previously, the area had been investigated during wide searches in the Orion complex with prismobjective and Schmidt plates, detecting a wealth of emission stars (e.g. Haro \& Moreno 1953; Wiramihardja et al. 1989), but the cluster had not been treated as an independent entity within the complex. Complete compilations of the determinations in the

\footnotetext{
* Tables A.1 to A.7 are only available in electronic form at the CDS via anonymous ftp to

cdsarc.u-strasbg.fr (130.79.128.5) or via

http://cdsweb.u-strasbg.fr/cgi-bin/qcat?]/A+A/478/667

$\star \star$ Formerly: Alexander von Humboldt Fellow at the Max-PlanckInstitut für Astronomie. Currently: Investigador Juan de la Cierva at the Universidad Complutense de Madrid.
}

literature of the age, heliocentric distance, frequency of discs and mass function of the $\sigma$ Orionis cluster are found in Caballero (2007a). A chapter of the Handbook of Star Forming Regions, edited by B. Reipurth, will be exclusively devoted to $\sigma$ Orionis (Walter et al., in press).

After the seminal work by Béjar et al. (1999), who found for the first time a rich population of young brown dwarfs in $\sigma$ Orionis, the cluster has turned into an excellent laboratory for the study of, e.g.:

- the search for free-floating planetary-mass objects (with masses below the deuterium-burning limit) and the study of the substellar mass function down to a few Jupiter masses (Zapatero Osorio et al. 2000; Béjar et al. 2001; González-García et al. 2006; Caballero et al. 2007);

- the frequency and the properties of $\sim 3$ Ma-old discs at different mass intervals (Jayawardhana et al. 2003; Oliveira et al. 2004, 2006; Hernández et al. 2007; Caballero et al. 2007; Zapatero Osorio et al. 2007a);

- the masses of OB-type stars in resolved binary systems (Heintz 1997; Mason et al. 1998; Caballero 2007d);

- the X-ray emission of young stars and brown dwarfs (Mokler \& Stelzer 2002; Sanz-Forcada et al. 2004; Franciosini et al. 2006; Caballero 2007b);

- the characterisitics of jets and Herbig-Haro objects (Reipurth et al. 1998; López-Martín et al. 2001; Andrews et al. 2004); and

- the photometric variability of low-mass stars and brown dwarfs (Bailer-Jones \& Mundt 2001; Caballero et al. 2004; Scholz \& Eislöffel 2004). 
Many interesting star-like objects have been discovered in the cluster, from the helium-rich, B2.0Vp-type magnetic star $\sigma$ Ori E (Greenstein \& Wallerstein 1958), through the Class I object candidate IRAS 05358-0238 (Oliveira \& van Loon 2004), to the hypothetical proplyd $\sigma$ Ori IRS1 (van Loon \& Oliveira 2003; Caballero 2005, 2007b). The most interesting objects in the cluster are, however, below the hydrogen-burning mass limit. Some of these substellar objects are the $\sim$ T6-type object S Ori 70 (which may be the least massive body directly detected out of the Solar System, $\sim 3 M_{\text {Jup }}$ - Zapatero Osorio et al. 2002c, 2007b; Burgasser et al. 2004), the T Tauri substellar analog S Ori J053825.4-024241 (which is the most variable brown dwarf yet found; Caballero et al. 2006a), the two strong $\mathrm{H} \alpha$ emitters at the planetary boundary S Ori 55 and S Ori 71 (with masses of only 10-20 $M_{\text {Jup }}$ and equivalent widths of the $\mathrm{H} \alpha$ line of up to $-700 \AA$; Zapatero Osorio et al. 2002b; Barrado y Navascués et al. 2002) and the brown dwarf-exoplanet system candidate SE $70+$ S Ori 68 (which could be the widest planetary system known so far; Caballero et al. 2006b). The number of known substellar objects in $\sigma$ Orionis is comparable to those of other rich, more massive, younger star-forming regions like Chamaeleon, Ophiuchus or the Orion Nebula Cluster. However, $\sigma$ Orionis is by far the region with the largest amount of brown dwarfs with membership confirmation ( $>30$; Caballero et al. 2007) and planetary-mass object candidates (29; Zapatero Osorio et al. 2000; González-García et al. 2006; Caballero 2007b; Caballero et al. 2007). Many of these bodies have measured L spectral types and/or flux excess longwards of $5 \mu \mathrm{m}$ (Zapatero Osorio et al. 2007a).

A lot of effort has been put into characterising the $\sigma$ Orionis cluster in general and investigating the connection between its stellar and substellar populations in particular (e.g. Béjar et al. 2004a; Sherry et al. 2004; Kenyon et al. 2005; Burningham et al. 2005; Caballero 2005, 2007a,b,c). The works by Kenyon et al. (2005), who investigated membership, binarity, and accretion among very low-mass stars and brown dwarfs surrounding $\sigma$ Ori, and, especially, Sherry et al. (2004) stand out. These authors presented an ambitious study, estimating the number of cluster members in the mass range $0.2 M_{\odot} \lesssim M \lesssim 1.0 M_{\odot}$ and the radius, age and total mass of the cluster. All these works are, however, incomplete or biased in some way: no comprehensive, homogenous, multi-band study, fully covering the whole stellar mass interval (from $\sim 20 M_{\odot}$ down to the substellar boundary) and the cluster area without gaps (from the very centre to the border) exists so far for $\sigma$ Orionis.

I extend the study of the brightest stars of the cluster shown in Caballero (2007a) down to the hydrogen-burning mass limit and beyond. I start out from a correlation between the 2MASS and DENIS catalogues within the environment of the Virual Observatory $^{1}$ and a bibliographic search of confirmed cluster members, and support them with spectroscopic and photometric data from the X-ray region to $120 \mu \mathrm{m}$ when available. The outcome of this work is the Mayrit catalogue, which comprises the majority of the stars and high-mass brown dwarfs of $\sigma$ Orionis.

\section{Analysis and results}

\subsection{The 2MASS/DENIS correlation}

The correlation between the Two-Micron All Sky Survey (2MASS) Catalog of Point Sources (Skrutskie et al. 2006) and the third release of the Deep Near Infrared Survey of the

\footnotetext{
${ }^{1}$ See http://www.ivoa.net
}

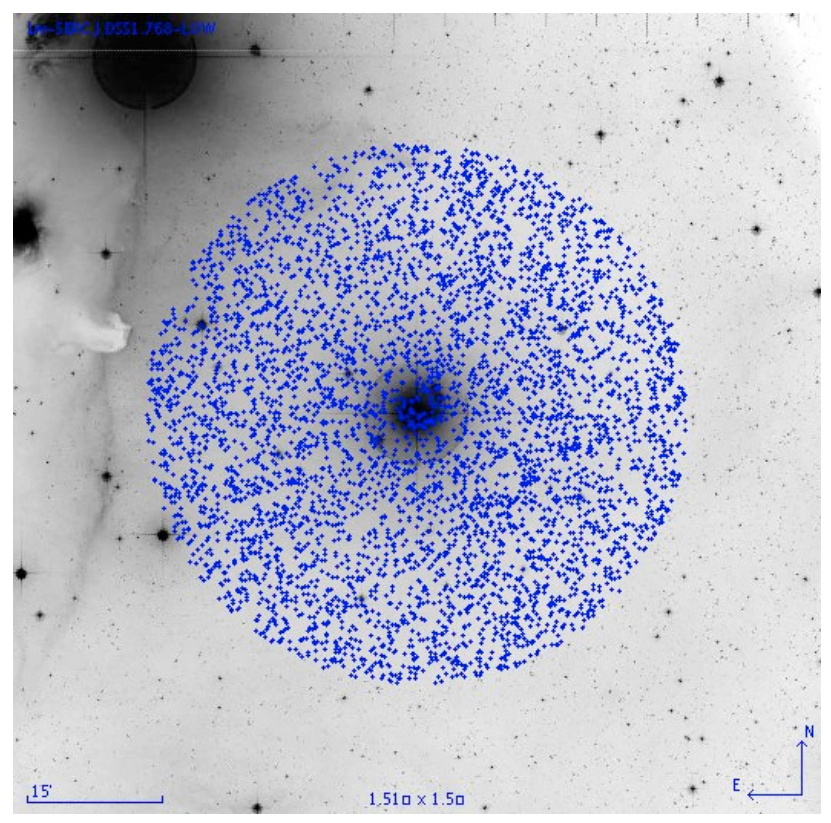

Fig. 1. Inverse-colour DSS-1 blue-band (photographip $B_{J}$ ) image centred in $\sigma$ Ori AB. Its size is $1.5 \times 1.5 \mathrm{deg}^{2}$. North is up and east is left. The correlated 2MASS/DENIS sources are marked with (blue) dots. Compare this figure with Fig. 1 in Caballero (2007a). Colour versions of all the figures are available in the electronic publication.
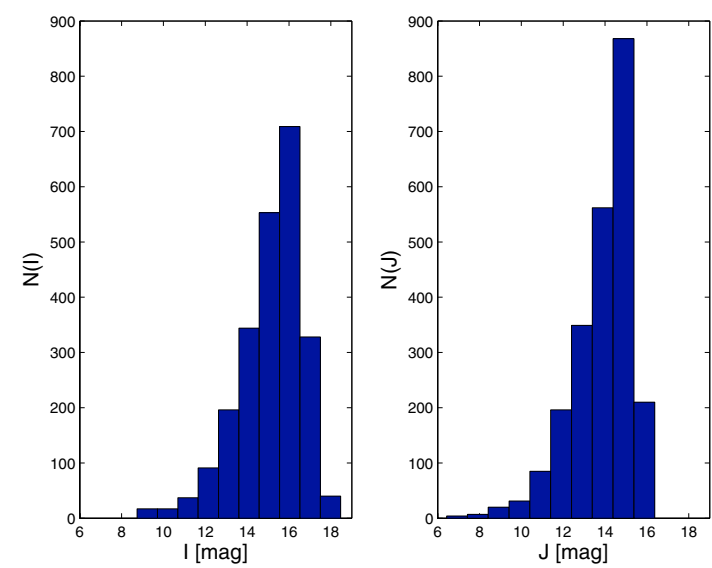

Fig. 2. Histograms of the number of sources as a function of DENIS $I$ (left) and 2MASS $J$ magnitudes (right).

Southern Sky (DENIS) database (Epchtein et al. 1997; DENIS Consortium 2005) were performed with the cross match tool of the Aladin sky atlas. I used a cross-match threshold of 1 arcsec, which is more than 10 times wider than the expected astrometric errors of the catalogues. The methodology and the survey area, a circle of 30 arcmin radius centred on $\sigma$ Ori $\mathrm{AB}$, are identical to those in Caballero (2007a), where further details can be found. The survey area and its surroundings are shown in Fig. 1.

Of the $57212 \mathrm{MASS}$ sources in the $2830 \mathrm{arcmin}^{2}$-wide area, $4951(\sim 87 \%)$ were correlated with DENIS sources. Most of the 770 non-correlated 2MASS sources are very faint and are expected to have fainter optical counterparts than the DENIS completeness. To avoid subsequent problems associated to high photometric uncertainties (e.g. faint blue sources with apparent red colours and vice versa), I only considered 2MASS sources with photometric errors $\delta K_{\mathrm{s}}<0.1 \mathrm{mag}$. With this restriction, the final sample size was 2332 optical/near-infrared sources. 
The histograms of the number of sources as a function of magnitude in the DENIS $I$ and 2MASS $J$ passbands are shown in Fig. 2. The bulk of the correlated sources have $I$ - and $J$-band magnitudes in the intervals $\sim 12-17 \mathrm{mag}$ and $\sim 11-16 \mathrm{mag}$, respectively. The number of sources peaks at $I \sim 16 \mathrm{mag}$ and $J \sim 15 \mathrm{mag}$ (and at $H \sim K_{\mathrm{s}} \sim 14 \mathrm{mag}$ ), which is expected from the increasing number of faint stars and the completeness and limiting magnitudes of the DENIS and 2MASS catalogues (DENIS $I_{5 \sigma}=18.0 \mathrm{mag} ; 2 \mathrm{MASS} J_{3 \sigma}=17.1 \mathrm{mag}, K_{\mathrm{s}, 3 \sigma}=$ $14.3 \mathrm{mag}$ ). Both surveys are complete at magnitudes brighter than $I \approx 16.5 \mathrm{mag}, J \approx 14.6 \mathrm{mag}$ and $K_{\mathrm{s}} \approx 12.8 \mathrm{mag}$, which are the magnitudes of the (non-reddened) faintest stars and brightest brown dwarfs in $\sigma$ Orionis (Caballero et al. 2007). Besides, 98\% $(91 \%)$ of the correlated sources have $I-J$ colours in the interval $0.4 \mathrm{mag} \lesssim I-J \lesssim 2.5 \mathrm{mag}(0.7 \mathrm{mag} \lesssim I-J \lesssim 2.1 \mathrm{mag})$, which are typical of normal dwarfs. Since the galactic latitude of the cluster is $b=-17.3 \mathrm{deg}$, a large contamination by red giants is not expected (Caballero et al., in prep.).

Other optical catalogues offered by the Virtual Observatory that could have been correlated with 2 MASS are, for example, the United States Naval Observatory USNO-B1.0 (Monet et al. 2003), the SuperCOSMOS Sky Survey SSS (Hambly et al. 2001), the Guide Star Catalogue ${ }^{2}$ GSC-II, or the Carlsberg Meridian Catalog ${ }^{3}$ CSC14. All of them are digitisations of Schmidt photographic plates. In spite of possible photometric uncertainties (see Sect. 2.3), the DENIS catalogue is deeper than they are. Besides, it is a CCD-based survey, which easily allows magnitudes to be transformed into fluxes and guarantees linearity within the dynamical range of the survey. Photographic magnitudes, in contrast, are not reliable at the faintest (especially GSC-II and CSC14) and brightest (especially SSS ${ }^{4}$ ) magnitudes. An optimum alternative to DENIS would be using USNO-B1 and SSS at the faintest and brightest magnitudes, respectively. This option still has a big drawback compared to DENIS (apart of a complex implementation): the incomplete coverage of the cluster centre due to the glare of $\sigma$ Ori AB. The inner 3 arcminradius circle contains about 40 cluster members and candidates and a large fraction of the cluster total mass (Caballero 2007a,b). On the one hand, DENIS tabulates optical data for all the sources with near-infrared magnitudes $J \lesssim 16.0$ mag from the outer radius of the survey at 30 arcmin to a few arcseconds to the Trapezium-like star system; more than 60 DENIS sources are in the central circle of radius $3 \mathrm{arcmin}$. On the other hand, USNO-B1 tabulates only three sources in the central circle (the OB-type stars $\sigma$ Ori $\mathrm{AB}, \mathrm{D}$, and E) and is complete and relatively free of glare artifacts only at more than $\sim 6$ arcmin. The effect of "glare incompleteness" is also detectable surrounding other bright early-type stars in the area with known nearby companions (e.g. HD 294271; Caballero 2005).

There are other non-virtual observatory optical data covering the $\sigma$ Orionis region, but they are not available to the author (e.g. Sherry et al. 2004), are too deep (i.e. all high- and intermediatemass stars are saturating; e.g. Béjar et al. 2004a,b), or avoided on purpose to survey the cluster centre (e.g. Caballero 2006). To sum up, the DENIS/MASS combination provides the most

${ }^{2}$ Space Telescope Science Institute and Osservatorio Astronomico di Torino.

${ }^{3}$ Copenhagen University Obs., Institute of Astronomy, Cambridge, UK, and Real Instituto y Observatorio de la Armada en San Fernando.

${ }^{4}$ The SSS/2MASS correlation gives rather blue optical/near-infrared colours, by up to $2 \mathrm{mag}\left(I_{\mathrm{SSS}}-K_{\mathrm{s}}\right)$, with respect to observational (Bessel \& Brett 1988) and theoretical (Schaller et al. 1992; Baraffe et al. 1998; Girardi et al. 2000) star sequences for all objects brighter than $I \approx 14$ mag. suitable optical/near-infrared correlation in the area. This combination is, nevertheless, incomplete for magnitudes brighter than $I \sim 6$ mag and offers incorrect magnitudes of objects brighter than $I \approx 10$ mag due to saturation and non-linear effects. To enhance the optical data from the DENIS catalogue, I have collected the $I$ magnitudes of the 18 brightest stars in the area from the USNO-B1 catalogue and from Caballero (2007b). Likewise, the 2MASS $J$ and $H$ photometry of $\sigma$ Ori AB is also affected by saturation, so I have used the respective values provided by Johnson et al. (1966). These incorporations led to simultaneously investigating in the survey all the sources with $J$-band magnitudes in the range $4.5-15.5 \mathrm{mag}$. Therefore, all the cluster stars and many of the high-mass brown dwarfs can be identified in the optical/near-infrared data.

\subsection{Membership classification}

\subsubsection{Known cluster members}

As a first step in the cluster member selection, a list of confirmed cluster members from the literature with a 2MASS/DENIS counterpart was made up, given in Table A.1. A confirmed cluster member must display at least one of the following features of youth, characteristic of $~ 3$ Ma-old stars or brown dwarfs:

- early spectral type (“OB”): O-, B-, and early-A-type stars taken from Caballero (2007a);

- Li I $\lambda 6707.8 \AA$ in absorption ("Li I"): mid- and late-type pre-main sequence stars mostly taken from Wolk (1996), Zapatero Osorio et al. (2002a), Kenyon et al. (2005), and Caballero (2006);

- strong and/or broad $\mathrm{H} \alpha \lambda 6562.8 \AA$ line in emission ("H $\alpha$ "): accretors and emission stars and brown dwarfs mostly taken from Haro \& Moreno (1953; Haro objects), Wiramihardja et al. (1989, 1991; Kiso objects), Zapatero Osorio et al. (2002a), Weaver \& Babcock (2004), and Caballero (2006);

- features of low-gravity ("low $g$ "): stars and brown dwarfs with weak alkali absorption lines (in particular, the $\mathrm{Na}$ I $\lambda \lambda 8183,8195 \AA$ A doublet) taken from Burningham et al. (2005);

- spectral energy distribution characteristic of objects with discs ("Class I", "II", "trans. disc", “ev. disc", "mIR"): Class I and Class II objects and stars with evolved or transition discs mostly taken from Oliveira \& van Loon (2004) and Hernández et al. (2007);

- very strong X-ray emission (“XX”, “XXX”): optical/nearinfrared counterparts of: (i) X-ray sources with count rates in the intervals $0.01-0.10 \mathrm{~s}^{-1}$ ("XX") or $>0.10 \mathrm{~s}^{-1}$ ("XXX") in the EPIC/XMM-Newton observations by Franciosini et al. (2006), or of (ii) X-ray sources out of the EPIC/XMM-Newton survey area detected by at least two independent surveys carried out with the space observatories Einstein, ASCA and ROSAT and with count rates $>0.01 \mathrm{~s}^{-1}$ in the WGACAT/ROSAT catalogue, mostly taken from Harris et al. (1994), Nakano et al. (1999) and White et al. (2000).

Table A.1 provides the Mayrit identifications of the 241 confirmed young stars and brown dwarfs in $\sigma$ Orionis that I was able to identify among the correlated 2MASS/DENIS sources. It also gives some of their alternative names from the literature, their features of youth, and corresponding abbreviated bibliographic references. The complete reference list is given in Table A.7. Some additional features of youth are also indicated in Table A.1 ("Em.", "Ca II": emission lines different from $\mathrm{H} \alpha$; "Si": silicon features in mid-infrared spectra, characteristic 
of dust coagulation in protoplanetary discs; "debris disc": flux excess detected only at the $24 \mu \mathrm{m}$ passband of MIPS/Spitzer; "X-flare": flares detected at $0.2-2.0 \mathrm{keV}$; "jet": central source associated to a Herbig-Haro object; "nebular": nebular appearence of the $\mathrm{H} \alpha$ emission; "X": stars with EPIC/XMM-Newton count rates in the interval $0.0005-0.01 \mathrm{~s}^{-1}$ ). Some marginally-detected features are marked with a question mark. Of the 241 identified cluster members, 187 have at least two known features of youth (and 95 have three or more features). Stars and brown dwarf candidates with only spectral-type determination from low-resolution spectroscopy have not been considered as confirmed cluster members.

There are also several cluster member candidates, like Kiso A-904 69 ([WB2004] 17), Haro 5-28 (Kiso A-0904 89), IRAS 05352-0227, or Haro 5-24, with published strong $\mathrm{H} \alpha$ emission and magnitudes that would make them detectable in this survey. However, because of the large errors in their coordinates, I was not able to identify them.

The Mayrit designation of the $\sigma$ Orionis cluster members and candidates used throughout this work follows the nomenclature introduced by Caballero (2007b).

\subsubsection{Fore- and background stars and galaxies}

To complement the list of known cluster members, I compiled 85 non-cluster member stars and 18 galaxies among the correlated 2MASS/DENIS sources, shown in Table A.2. Nine stars are presented here for the first time, while the remaining noncluster members were taken from the literature. In particular, these stars are:

- bright stars with high tangential velocity and K- and M-type foreground stars taken from Caballero (2007a); and

- stars whose high-quality spectra show no lithium in absorption ("no Li I"), no low-gravity features in the alkali lines ("no low $g$ "), and/or discordant radial velocity with respect to the cluster systemic radial velocity ("no $\mathrm{V}_{r}$ "), taken from Kenyon et al. (2005), Burningham et al. (2005), and Caballero (2006).

I also got the proper motion tabulated by USNO-B1 (very similar to those tabulated by SSS and NOMAD1 - Zacharias et al. 2004) for all the correlated 2MASS/DENIS sources brighter than $I=12.5 \mathrm{mag}$ that were not investigated in the Tycho-2/2MASS correlation by Caballero (2007a). I estimate that the average error of the USNO-B1 measurements in the investigated magnitude interval is $\delta \mu \gtrsim 5 \mathrm{mas} \mathrm{a}^{-1}$. There are, however, some stars with relatively high proper motions and larger uncertainties (e.g. SO430116, with the second highest proper motion in the area after G 99-20, has a SSS $\mu=(+103 \pm 5$, $-123 \pm 5)$ mas a $^{-1}$, while USNO-B1 tabulates a proper motion $\sim 30 \%$ less). Of the 85 non-cluster members, 29 have propermotion moduli larger than $10 \mathrm{mas} \mathrm{a}^{-1}$, which was the value used to separate young stars and candidate Ori OB $1 \mathrm{~b}$ association members from probable foreground stars with higher tangential velocities in Caballero (2007a). The proper-motion diagram shown in Fig. 3 illustrates the study of the star tangential velocities. Seven of the nine new non-cluster members first listed here have proper motions $\mu \gtrsim 20$ mas a $^{-1}$. The remaining two new non-cluster members, with proper motions consistent with membership in $\sigma$ Orionis, probably are foreground $\mathrm{K}$ - or early-M-type stars because they have very red colours, but they are much brighter than cluster members of a similar expected spectral type and do not show any characteristic feature of $\mathrm{T}$ Tauri stars. These two stars

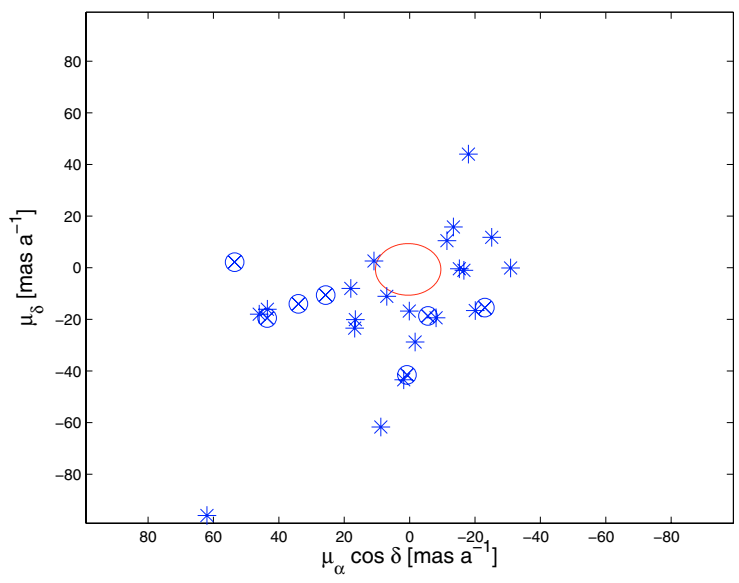

Fig. 3. Proper-motion diagram $\left(\mu_{\delta}\right.$ vs. $\mu_{\alpha} \cos \delta$ ) of foreground stars with $\mu>10$ mas a $^{-1}$. Non-cluster members from the literature and new noncluster members from this work are shown with asterisks (“*”) and tensorial product symbols (" $\otimes$ "), respectively. The threshold used by Caballero (2007a) to separate Ori OB $1 \mathrm{~b}$ members from stars with high tangential velocities is marked with a big ellipse. The high-propermotion star G 99-20 (LP 598-162; $\mu=288$ mas a $^{-1}$ ) is not shown for the sake of clarity.

are 2MASS J05400217-0253423 $\left(I=10.97 \pm 0.06 \mathrm{mag}, K_{\mathrm{s}}=\right.$ $7.74 \pm 0.03 \mathrm{mag})$ and 2MASS J05390143-0253431 ( $I=11.28 \pm$ $0.03 \mathrm{mag}, K_{\mathrm{s}}=8.40 \pm 0.03 \mathrm{mag}$ ). Table A. 2 gives the $2 \mathrm{MASS}$ designations of all the fore- and background stars, alternative names if they exist, a flag indicating if they have been spectroscopically studied ("Sp." = Yes/No), their USNO-B1 proper motions if they are larger than 10 mas a $^{-1}$, remarks and abbreviated references (see again Table A.7 for complete references). The nine new non-cluster members have a blank in the reference column.

In Table A.3, I list the 2MASS designations of 18 galaxies found in the survey area. All except one (2E 1448, which is a very strong X-ray emitter; Caballero \& López-Santiago, in prep.) are tabulated in the Final Release of 2MASS Extended Sources (2MASS 2003). Some of these galaxies were also identified in the Spitzer Space Telescope study in $\sigma$ Orionis by Hernández et al. (2007). As an additional test, I checked the extended $F W H M$ s of the ten reddest galaxies $\left(J-K_{\mathrm{s}} \gtrsim 1.4 \mathrm{mag}\right)$ using the SSS digitisations.

\subsubsection{Candidate young stars and brown dwarfs}

I have selected 109 cluster member candidates without membership information based on their position with respect to the 241 confirmed cluster members in the $I$ vs. $I-K_{\mathrm{s}}$ colourmagnitude diagram shown in Fig. 4. The cluster members with known features of youth define a quite broad photometric sequence in the diagram. I classified as candidate cluster stars and brown dwarfs those correlated 2MASS/DENIS sources that are neither known cluster members (Sect. 2.2.1) nor non-cluster members (Sect. 2.2.2) and that fall redwards of the solid line shown in Fig. 4 or are brighter than $I=11.5 \mathrm{mag}$. The solid line leaves redwards of it $\sim 75 \%$ of the confirmed cluster members. All the stars in the area brighter than $I=9.5$ mag are confirmed cluster members.

Figure 1 shows that the survey area partially overlaps with a nebulosity that forms an extension of the Orion B cloud associated to the Alnitak-Horsehead Nebula-IC 434-Flame Nebula complex $($ Alnitak $=\zeta$ Ori). Twelve candidate cluster members 


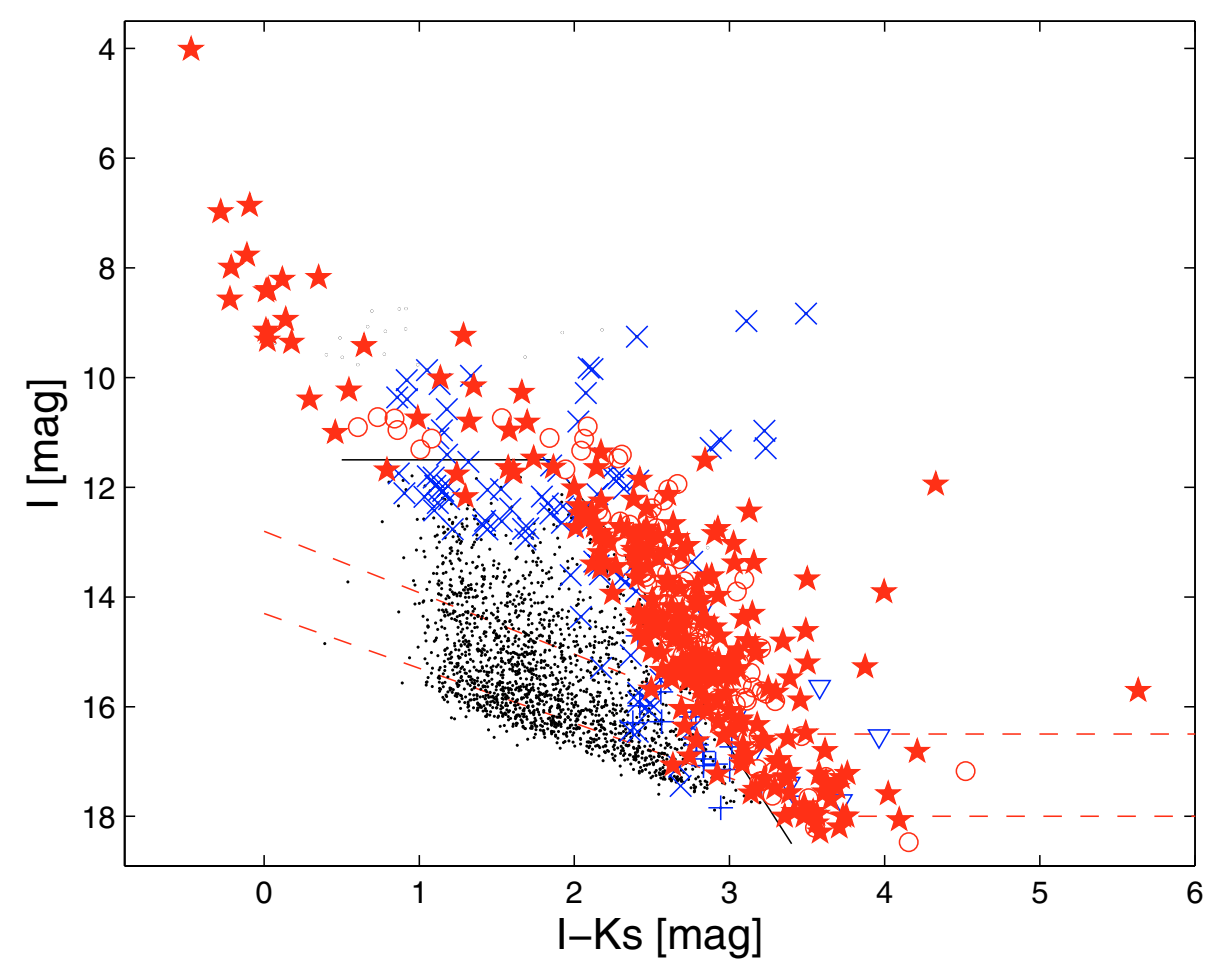

Fig. 4. $I$ vs. $I-K_{\mathrm{s}}$ colour-magnitude diagram in the survey area. Filled (red) stars: stars and brown dwarfs with known features of youth from the literature; open (red) circles: photometric candidate cluster stars and brown dwarfs; (blue) pluses: galaxies; open (blue) down triangles: reddened sources in the northeastern cloud; (blue) x-marks: fore- and background stars; tiny (black) dots: unresolved galaxies, probable fore- and background stars and cluster stars and brown dwarfs with blue $I-K_{\mathrm{s}}$ colours for their $I$ magnitudes; solid (black) line: criterion for selecting candidate cluster stars and brown dwarfs without known features of youth; dashed (red) lines: approximate completeness and detection limits of the survey.

to the northeast of the survey area, together with three known non-cluster members with spectroscopic information, fall exactly below the densest (filament-shaped) part of the nebulosity. This area is shown in detail in Fig. 6 (see also the upper part of Fig. 2 in Hernández et al. 2007). The cloud shred also coincides with an alignment of mid-infrared sources in the IRAS Catalogue of Point Sources and in the IRAS Serendipitous Survey Catalog without known optical or near-infrared counterparts (IPAC 1986; also in the IRAS Point Source Reject Catalog). Although some of these sources might be bona-fide, embedded young sources associated to the filament, I list them in Table A.4 as probable reddened sources that do not belong to the $\sigma$ Orionis cluster. Three of them were also photometric cluster member candidates in the work by Sherry et al. (2004). Besides, Béjar et al. (2004a) proposed that an overdensity of IZ-band photometric cluster members to the northeast of $\sigma$ Orionis could be associated to a hypothetical cluster surrounding Alnitak. The existence of the nebulosity adds further complexity to the topic. The solution to this dilemma requires more spectroscopy.

After taking into account the 109 sources redwards of the solid line in Fig. 4 and the 12 probable reddened sources in Table A.4, then 97 reliable candidate cluster members without membership information, of which 40 are new, remain from the survey. Their designations and alternative names, together with remarks and references, are provided in Table A.5. Remarks column indicates, if known, spectral type, the period of photometric variability and X-ray emission ("X": count rate in the interval $0.0005-0.01 \mathrm{~s}^{-1}$ in Franciosini et al. 2006; "X?": source possibly associated to X-ray events in the WGACAT/ROSAT catalogue by White et al. 2000). The marginal detections of youth features ( $\mathrm{Li} \mathrm{I}, \mathrm{H} \alpha$, low $g$ ) are indicated with a question mark.
The pictogramme of the spatial distribution of confirmed cluster members, non-cluster members, galaxies, and candidate cluster members is displayed in Fig. 5. Since most of the spectroscopic, mid-infrared and X-ray follow-ups are focused on the cluster centre, photometric candidate cluster members do not concentrate towards the cluster centre as sharply as confirmed cluster members do ${ }^{5}$.

\subsection{Refinement of optical photometry}

As a final step in the analysis, I improved the DENIS $I$ data of the correlated sources. The DENIS database sometimes tabulates two or more detections separated by only $\sim 0.1-0.3 \operatorname{arcsec}$ with slightly different magnitudes. A visual inspection shows that the detections are associated to the same source. Often, one or some detections have very large photometric uncertainties, of up to $\delta I=1.00 \mathrm{mag}$. During the the $2 \mathrm{MASS} / \mathrm{DENIS}$ cross match, only one DENIS source (the nearest one to the 2MASS counterpart) was correlated, which may have larger photometric errors than the other sources. Therefore, I investigated the possible multiple detections in the DENIS database of the 241 confirmed cluster members and the 97 candidate cluster members. Of them, 55 sources $(\sim 16 \%)$ have such multiple (double) detections, $I_{1}$ and $I_{2}$.

\footnotetext{
${ }^{5}$ Caballero (2007c) used a previous version of the Mayrit catalogue to investigate the spatial distribution of stars and brown dwarfs in $\sigma$ Orionis. The only difference was that the X-ray galaxy 2E 1456 was considered as a photometric cluster member candidate. Even accounting for this difference, the results of that paper (clustering parameter $Q \approx 0.88$, volume density $\rho(r) \propto r^{-2}$, mass-dependent radial distribution, azimuthal asymmetry) remain unchanged.
} 


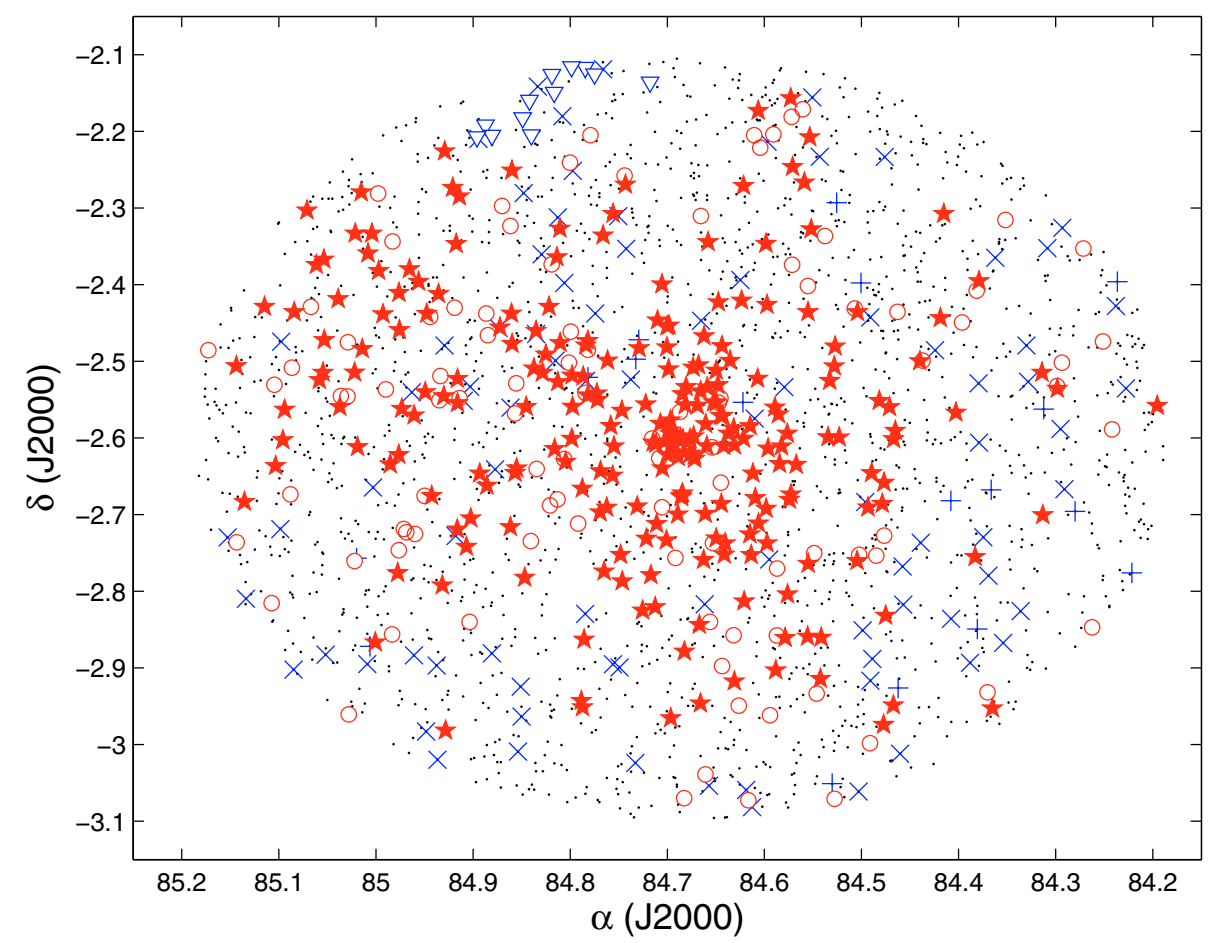

Fig. 5. Spatial distribution of the investigated sources. The symbol code is as in Fig. 4.

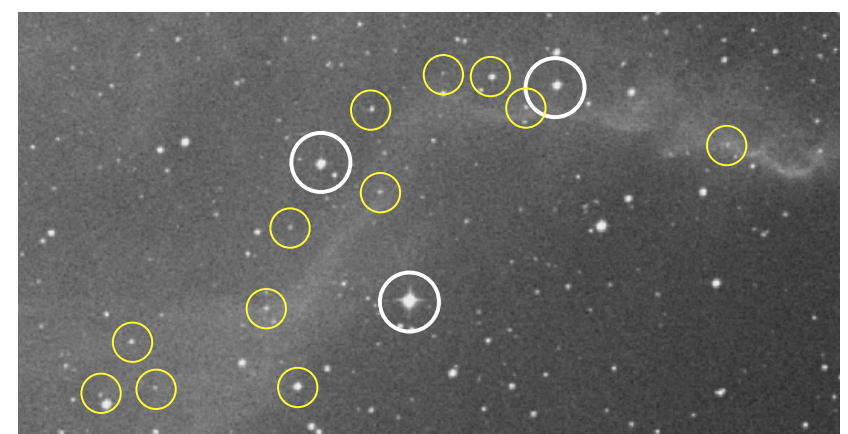

Fig. 6. Part of the ESO Red (photographic $R$ ) image of the northeastern nebulosity discussed in the text. The three known non-cluster members are marked with big thick (white) circles. The 12 reddened sources previously considered as candidate cluster members are marked with small thin (yellow) circles. The gas emission is due to $\mathrm{H} \alpha$ (mostly) and [N II] $\lambda \lambda 6548.0,6583.4 \AA$. North is up and east is the left; approximate size is $17 \times 7 \operatorname{arcmin}^{2}$.

The difference between the two tabulated I-band magnitudes, $I_{1}$ and $I_{2}$, as a function of the most accurate measurement is shown in Fig. 7. While the mean of the quantity $\left(\delta^{2} I_{1}+\delta^{2} I_{2}\right)^{1 / 2}$, which measures the combined photometric uncertainties, is $0.56 \mathrm{mag}$, the standard deviation of the difference $I_{1}-I_{2}$ is only $0.26 \mathrm{mag}$. There are only three objects with $I_{1}-I_{2}>0.56 \mathrm{mag}$ (V603 Ori, Haro5-18 and SWW 34), of which two are known to be intense accretors and one is known to be a photometric variable (Haro \& Moreno 1953; Salukvadze 1987; Wiramihardja et al. 1989; Andrews et al. 2004). These values must be compared with typical uncertainties of normalised magnitudes from plate digitisations, of no less than $\sim 0.3 \mathrm{mag}$ (Hambly et al. 2001). These facts indicate that $(i)$ the large DENIS photometric uncertainties in the optical (especially $\delta I=1.00 \mathrm{mag}$ ) are overestimated, and (ii) the uncorrected DENIS magnitudes can still be used with uncertainties at the 0.26 mag level, except for

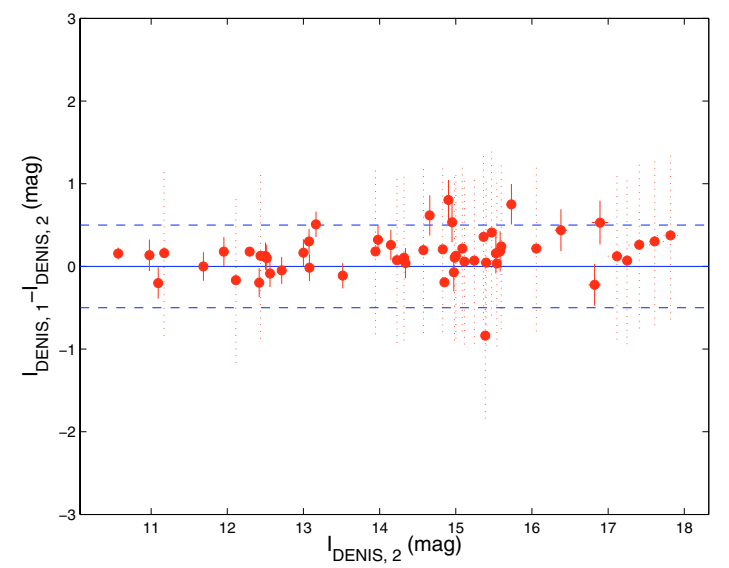

Fig. 7. $I_{1}-I_{2}$ vs. $I_{2}$ of stars with double detections in the DENIS database. The errorbars associated to stars with photometric uncertainties $\delta I=1.00 \mathrm{mag}$ are plotted with dotted lines. Horizontal lines denote $I_{1}-I_{2}=0.0 \mathrm{mag}$ (solid) and $I_{1}-I_{2}= \pm 0.5 \mathrm{mag}$ (dashed).

high-amplitude variables. The latter objects have, however, extremely red $J-K_{\mathrm{s}}$ colours (of $2.14 \pm 0.03 \mathrm{mag}$ in the case of V603 Ori) and are easy to recognise among fore- and background sources. Therefore, the DENIS multiple detections barely affect my selection criterion.

\subsection{The Mayrit catalogue}

Finally, Table A.6 tabulates the Mayrit designations, the most accurate DENIS $I$-band and 2MASS $J H K_{\mathrm{S}}$ magnitudes, and J2000 coordinates of the 338 cluster members and member candidates that constitute the Mayrit catalogue. The last column indicates with a filled star (" $\star$ ") if the object is a confirmed cluster member with at least one known feature of youth. Of the 338 objects, only 16 have no cluster membership information and are fainter than the completeness of the search; likewise, only 3 member 
candidates are fainter than the detection limit. However, the numbers of confirmed cluster members fainter than the completeness (43) and detection (15) limits are larger. The correspondingly larger photometric uncertainties might explain the width of the bottom of the cluster sequence.

The catalogue has advantages and disadvantages. On the one hand, its most noticeable pros are:

- comprehensiveness: tabulated Mayrit sources outnumbers previous studies in $\sigma$ Orionis;

- very wide investigated magnitude interval: it translates into a very wide mass interval that ranges four orders of magnitude, from the $18+12 M_{\odot}$ of $\sigma$ Ori $\mathrm{AB}$ to the $\sim 0.03 M_{\odot}$ of B05 2.03-671 (Caballero \& Chabrier, in prep.). The cluster stellar and substellar populations had been traditionally studied in mass bits;

- continuity and symmetry of the survey area: in contrast to previous searches in the cluster, there are no gaps between detectors or asymmetric layouts.

- data homogeneity: only DENIS and 2MASS astrometric and photometric data are tabulated for all the objects (except for a few bright stars - see Sect. 2.1);

On the other hand, some disadvantages of the Mayrit catalogue are:

- extreme heterogenity of the list of confirmed cluster members: I examined several dozens works, including spectroscopy, mid-infrared photometry, and X-ray emission, for making up the catalogue;

- moderate size $\left(0.78 \mathrm{deg}^{2}\right)$ : a search radius larger than 30 arcmin would lead to surveying the Horsehead NebulaIC 434 complex, where the extinction is very high and background objects are reddened (just as in Sect. 2.2.3). The variable extinction would make the cluster member selection very difficult;

- incompleteness and contamination, which depend on the brightness interval and the spatial distribution of young stars in the Ori OB $1 \mathrm{~b}$ association. Both incompleteness and contamination of this catalogue will be discussed in a future work.

\section{Summary}

The $\sim 3$ Ma-old $\sigma$ Orionis cluster is a new cornerstone for observational and theoretical studies with the aim of understanding the general processes of collapse and fragmentation of a molecular cloud, formation of stars and substellar objects, and evolution of circumstellar discs. I present a comprehensive, fairly complete catalogue of cluster members that can be used for further studies in $\sigma$ Orionis (e.g. spatial distribution, multiplicity, mass function and frequency, and characterisation of discs). This investigation covers the whole stellar and part of the brown dwarf domain of the cluster from $\sim 18$ to $\sim 0.03 M_{\odot}$.

I performed an $I K_{\mathrm{S}}$ survey in a 30 arcmin-radius region centred on the $\mathrm{O} 9.5 \mathrm{~V}+\mathrm{B} 0.5 \mathrm{~V}$ binary $\sigma$ Ori $\mathrm{AB}$ using the Aladin tool and optical and near-infrared data from the DENIS and 2MASS catalogues. The photometric data were complemented with information from the literature regarding the membership of known sources. I have compiled a list of 241 cluster stars and brown dwarfs with known features of youth (e.g. early spectral types, Li I in absorption, near and mid infrared excess attributed to surrounding discs, strong X-ray, and $\mathrm{H} \alpha$ emissions), 85 foreand background stars with astrometric and spectroscopic data (nine are new), 18 galaxies with extended $F W H M$ s, and 12 probable reddened sources in a nebulosity northeaster of the survey area, associated to the Horsehead Nebula. From the $I$ vs. $I-K_{\mathrm{s}}$ diagram, I have selected 97 additional photometric cluster member candidates without reliable membership information. This makes a list of $338 \sigma$ Orionis members and member candidates, the Mayrit catalogue, of which more than $70 \%$ display features of extreme youth. I tabulate precise coordinates, $I J H K_{\mathrm{s}}$, and supplementary information of all the cluster members, member candidates and non-members.

Acknowledgements. I thank the anonymous referee, J. Adams, C. A. L. Bailer-Jones, T. Forveille and, R. Mundt for helpful suggestions and discussion. Partial financial support was provided by the Universidad Complutense de Madrid and the Spanish Ministerio Educación y Ciencia under grants AyA2004-00253 and AyA2005-02750 of the Programa Nacional de Astronomía y Astrofísica and by the Comunidad Autónoma de Madrid under PRICIT project S-0505/ESP-0237 (AstroCAM).

\section{References}

Alcalá, J. M., Terranegra, L., Wichmann, R., et al. 1996, A\&AS, 119, 7

Andrews, S. M., Reipurth Bo, Bally, J., \& Heathcote, S. R. 2004, ApJ, 606, 353 Bailer-Jones, C. A. L., \& Mundt, R. 2001, A\&A, 367, 218

Barrado y Navascués, D., Zapatero Osorio, M. R., Martín, E. L., et al. 2002, A\&A, 393, L85

Barrado y Navascués, D., Béjar, V. J. S., Mundt, R., et al. 2003, A\&A, 404, 171 Béjar, V. J. S., Zapatero Osorio, M. R., \& Rebolo, R. 1999, ApJ, 521, 671

Béjar, V. J. S., Martín, E. L., Zapatero Osorio, M. R., et al. 2001, ApJ, 556, 830

Béjar, V. J. S., Caballero, J. A., Rebolo, R., Zapatero Osorio, M. R., \& Barrado y Navascués, D. 2004a, Ap\&SS, 292, 339

Béjar, V. J. S., Zapatero Osorio, M. R., \& Rebolo, R. 2004b, AN, 325, 705

Brown, A. G. A., de Geus, E. J., \& de Zeeuw, P. T. 1994, A\&A, 289, 101

Burgasser, A. J., Kirkpatrick, J. D., McGovern, M. R., et al. 2004, ApJ, 604, 827

Burningham, B., Naylor, T., Littlefair, S. P., \& Jeffries, R. D. 2005, MNRAS, 356,1583

Caballero, J. A. 2005, AN, 326, 1007

Caballero, J. A. 2006, Ph.D. Thesis, Universidad de La Laguna

Caballero, J. A. 2007a, A\&A, 466, 917

Caballero, J. A. 2007b, AN, 328, 917

Caballero, J. A. 2007c, MNRAS, in press, [arXiv:0710.1255], doi:10.1111/j.1365-2966.2007.12555.x

Caballero, J. A., Béjar, V. J. S., Rebolo, R., \& Zapatero Osorio, M. R. 2004, A\&A, 424, 857

Caballero, J. A., Martín, E. L., Zapatero Osorio, M. R., et al. 2006a, A\&A, 445, 143

Caballero, J. A., Martín, E. L., Dobbie, P. D., \& Barrado y Navascués, D. 2006b, A\&A, 460, 635

Caballero, J. A., Béjar, V. J. S., Rebolo, R., et al. 2007, A\&A, 470, 903

Cannon, A. J., \& Pickering, E. C. 1918, AnHar, 92, 1

Catalano, F. A., \& Renson, P. 1998, A\&AS, 127, 421

Cunha, K., Smith, V. V., \& Lambert, D. L. 1995, ApJ, 452, 634

Downes, R. A., \& Keyes, C. D. E. 1988, AJ, 96, 777

Epchtein, N., de Batz, B., Capoani, L., et al. 1997, Msngr, 87, 27

Franciosini, E., Pallavicini, R., \& Sanz-Forcada, J. 2006, A\&A, 446, 501

Frost, E. B., \& Adams, W. S. 1904, ApJ, 19, 151

García-Lario, P., Manchado, A., Suso, S. R., Pottasch, S. R., \& Olling, R. 1990, A\&AS, 82, 497

Garrison, R. F. 1967, PASP, 79, 433

Giclas, H. L., Burnham, R., \& Thomas, N. G. 1961, Bulletin/Lowell Observatory; Bulletin (Lowell Observatory); No. 112 (Flagstaff, Ariz.: Lowell Observatory), 61

González-García, B. M., Zapatero Osorio, M. R., Béjar, V. J. S., et al. 2006, A\&A, 460, 799

Gray, R. O., \& Corbally, C. J. 1993, AJ, 106, 632

Greenstein, J. L., \& Wallerstein, G. 1958, ApJ, 127, 237

Grillo, F., Sciortino, S., Micela, G., Vaiana, G. S., \& Harnden, F. R., Jr. 1992, ApJS, 81, 795

Guetter, H. H. 1981, AJ, 86, 1057

Hambly, N. C., MacGillivray, H. T., Read, M. A., et al. 2001, MNRAS, 326, 1279

Haro, G., \& Moreno, A. 1953, BOTT, 1g, 11

Harris, D. E., Stern Grant, C. P., \& Andernach, H. 1994, IAUJD, 20, 277

Heintz, W. D. 1997, ApJS, 111, 335

Herbig, G. H., \& Kameswara Rao, N. 1972, ApJ, 174, 401

Hernández, J., Hartmann, L., Megeath, T., et al. 2007, ApJ, 662, 1067 
Hirth, G. A., Mundt, R. \& Solf, J. 1997, A\&AS, 126, 437

Hunger, K., Heber, U., \& Groote, D. 1989, A\&A, 224, 57

Jayawardhana, R., Ardila, D. R., \& Stelzer, B. 2003, Brown Dwarfs, Proc. IAU Symp. 211, held 20-24 May 2002 at University of Hawai'i, Honolulu, Hawai'i. ed. E. L. Martín (San Francisco: ASP), 139

Johnson, H. L., Iriarte, B., Mitchell, R. I., \& Wisniewskj, W. Z. 1966, CoLPL, 4 99

Joncas, G., \& Borra, E. F. 1981, A\&A, 94, 134

Kenyon, M. J., Jeffries, R. D., Naylor, T., Oliveira, J. M., \& Maxted, P. F. L. 2005, MNRAS, 356, 89

Lee, T. A. 1968 , ApJ, 152, 913

Lee, C. W., Martín, E. L., \& Mathieu, R. D. 1994, AJ, 108, 1445

Lesh, J. R. 1968, ApJ, 152, 905

van Loon, J. Th., \& Oliveira, J. M. 2003, A\&A, 405, L33

López-Martín, L., Raga, A. C., López, J. A., \& Meaburn, J. 2001, A\&A, 371, 1118

Lyngå, G. 1981, The Catalogue of Open Star Clusters, ADCBu, 1, 90

Mason, B. D., Gies, D. R., Hartkopf, W. I., et al. 1998, AJ, 115, 821

Monet, D. G., Levine, S. E., Casian, B., et al. 2003, AJ, 125, 984

Moran, E. C., Helfand, D. J., Becker, R. H., White, R. L. 1996, ApJ, 461, 127

Morgan, W. W., Keenan, P. C., \& Kellman, E. 1943, An atlas of stellar spectra, with an outline of spectral classification, Chicago, Ill (The University of Chicago press)

Muzerolle, J., Hillenbrand, L., Calvet, N., Briceño, C., \& Hartmann, L. 2003, ApJ, 592, 266

Nakano, M., Yamauchi, S., Sugitani, K., Ogura, K., \& Kogure, T. 1999, PASJ, 51,1

Nesterov, V. V., Kuzmin, A. V., Ashimbaeva, N. T., et al. 1995, A\&AS, 110, 367

Neubauer, F. J. 1943, ApJ, 97, 300

Nissen, P. E. 1976, A\&A, 50, 343

North, P. 1984, A\&AS, 55, 259

Oliveira, J. M., \& van Loon, J. Th. 2004, A\&A, 418, 663

Oliveira, J. M., Jeffries, R. D., Kenyon, M. J., Thompson, S. A., \& Naylor, T. 2002, A\&A, 382, L22

Oliveira, J. M., Jeffries, J. D., \& van Loon, J. Th. 2004, MNRAS, 347, 1327

Oliveira, J. M., Jeffries, R. D., van Loon, J. Th., \& Rushton, M. T. 2006, MNRAS, 369, 272
Reipurth Bo, B. J., Fesen, R. A., \& Devine, D. 1998, Nature, 396, 343 Renson, P. 1998, A\&AS, 76, 127

Salukvadze, N. 1987, AbaOB, 62, 57

Sanduleak, N. 1971, PASP, 83, 95

Schild, R. E., \& Chaffee, F. 1971, ApJ, 169, 529

Scholz, A., \& Eislöffel, J. 2004, A\&A, 419, 249

Sherry, W. H., Walter, F. M., \& Wolk, S. J. 2004, AJ, 128, 2316

Skrutskie, M. F., Cutri, R. M., \& Stiening, R. 2006, AJ, 131, 1163

Stephenson, C. B. 1986, AJ, 92, 139

Tovmasyan, G. M., \& Oganesyan, R. Kh. 1991, Afz, 34, 301 Two Micron All Sky Survey (2MASS) Team, 2MASS Extended Objects, Final Release

Ueda, Y., Ishisaki, Y., Takahashi, T., Makishima, K., \& Ohashi, T. 2001, ApJS, 133, 1

Walter, F. M., Wolk, S. J., Freyberg, M., \& Schmitt, J. H. M. M. 1997, MmSAI, 68,1081

Warren, W. H. Jr., \& Hesser, J. E. 1978, ApJS, 36, 497

Weaver, Wm. B., \& Babcock, A. 2004, PASP, 116, 1035

White, N. E., Giommi, P., \& Angelini, L. 2001, VizieR On-line Data Catalog: IX/31. Originally published in: Laboratory for High Energy Astrophysics (LHEA/NASA), Greenbelt 2000

Wiramihardja, S. D., Kogure, T., Yoshida, S., Ogura, K., \& Nakano M. 1989, PASJ, 41, 155

Wiramihardja, S. D., Kogure, T., Yoshida, S., et al. 1991, PASJ, 43, 27

Wolk, S. J. 1996, Ph.D. Thesis, State Univ. New York at Stony Brook

Yamauchi, S., Kamimura, R., \& Koyama, Ka. 2000, PASJ, 52, 161

Zacharias, N., Monet, D. G., Levine, S. E., et al. 2004, American Astronomical Society Meeting 205, \#48.15; BAAS, 36, 1418

Zapatero Osorio, M. R., Béjar, V. J. S., Martín, E. L., et al. 2000, Science, 290, 103

Zapatero Osorio, M. R., Béjar, V. J. S., Pavlenko, Ya., et al. 2002a, A\&A, 384, 937

Zapatero Osorio, M. R., Béjar, V. J. S., Martín, E. L., Barrado y Navascués, D., \& Rebolo, R. 2002b, ApJ, 569, L99

Zapatero Osorio, M. R., Béjar, V. J. S., Martín, E. L., et al. 2002c, ApJ, 578, 536

Zapatero Osorio, M. R., Caballero, J. A., Béjar, V. J. S., et al. 2007, A\&A, 472, L9 